\title{
The impact of preschool wheezing patterns on health-related quality of life at age 4 years
}

\author{
Esther Hafkamp-de Groen*,\#, Ashna D. Mohangoo", Jeanne M. Landgraf ${ }^{+}$, Johan \\ C. de Jongste ${ }^{\S}$, Liesbeth Duijts ${ }^{\S, f}$, Henriëtte A. Moll**, Vincent W.V. Jaddoe ${ }^{\star, f, * \star}$, \\ Albert Hofman ${ }^{f}$ and Hein Raat ${ }^{\#}$
}

ABSTRACT: We assessed whether dynamic preschool wheezing patterns affect health-related quality of life (HRQOL) at age 4 years.

The study included 3878 children participating a prospective cohort study. Information on preschool wheezing was obtained by questionnaire and children were categorised into: never, early, late and persistent wheezing. At age 4 years HRQOL was measured using the Child Health Questionnaire (CHQ).

Persistent wheezing was associated with reduced scores for nine out of $13 \mathrm{CHQ}$ scales. No differences in psychosocial CHQ scores ( $p>0.05$ ), but lower physical CHQ scores were found in children with late and persistent wheezing, compared to children who never wheezed $(p<0.001)$. Mean scores on general health perceptions were, respectively, eight and 12 points lower (on a $0-$ 100 scale) in children with late and persistent wheezing $(p<0.001)$, and children with one to three episodes and four or more episodes of wheezing in the fourth year respectively scored seven and 24 points lower $(p<0.001)$, compared to children who never wheezed.

Persistent wheezing during preschool age independently affects a child's HRQOL, particularly general health perceptions and physical domains at age 4 years. HRQOL was more affected by frequent wheezing episodes in the fourth year of life, rather than by the duration of wheezing at age $0-4$ years.

KEYWORDS: Asthma symptoms, Child Health Questionnaire-Parent Form 28 items, preschool children, well-being, wheezing

W heezing is highly prevalent in children, especially in the first years of life. Wheezing is the most important symptom of asthma and is one of the leading causes of morbidity in early childhood [1]. Over the past decade, the use of health-related quality of life (HRQOL) as an essential outcome measure of asthma treatment and management has increased $[2,3]$. HRQOL assesses the functional impact of asthma symptoms across multiple clinically relevant domains. Ultimately, the goal of asthma management is to achieve both optimal disease control and HRQOL improvements [4, 5]. Recent findings suggest that clinical efforts to improve health outcomes in paediatric asthma should target those at risk for poor HRQOL [6].

Several studies have investigated the impact of asthma on children's HRQOL, focusing on the severity of asthma symptoms [7-10]. The majority of these studies have been cross-sectional [7-9].
The available evidence suggests an association between wheezing and HRQOL $[8,9]$, but the dynamics of how wheezing over-time affects children's HRQOL remains unclear. Wheezing symptoms are often non-specific, and might partly be due to respiratory tract infections. Cross-sectional studies on the association between preschool wheezing and HRQOL have been inconclusive.

It is important to understand the impact of wheezing patterns on HRQOL in preschoolers, because inadequate management of asthma in children between the ages of 2 and 8 years seems common [11]. We hypothesised that HRQOL is more likely to be impaired in preschool children with persistent wheezing, compared to children with transient or without wheezing.

The aim of our study was to assess whether dynamic preschool wheezing patterns affect a child's HRQOL at age 4 years, using the parent

\section{AFFILIATIONS}

*The Generation R Study Group, Erasmus Medical Centre, Rotterdam "Dept of Public Health, Erasmus Medical Centre, Rotterdam,

"TNO, Netherlands Organisation for Applied Scientific Research, Dept Child Health, Leiden,

${ }^{\text {s}}$ Dept of Paediatrics, Division of Respiratory Medicine, Erasmus Medical Centre-Sophia Children's Hospital, Rotterdam,

${ }^{f}$ Dept of Epidemiology, Erasmus Medical Centre, Rotterdam, and **Dept of Paediatrics, Erasmus Medical Centre-Sophia Children's Hospital, Rotterdam, The Netherlands.

${ }^{+}$Health Act $\mathrm{CHQ}$, Cambridge MA, USA.

CORRESPONDENCE

E. Hafkamp-de Groen

P0 Box 2040 (Room Ae-001)

3000 CA Rotterdam

The Netherlands

E-mail: e.hafkamp@erasmusmc.nl

Received:

Jan 252012

Accepted after revision:

June 172012

First published online: July 122012 
form of the Child Health Questionnaire (CHQ-PF28). In particular, we explored whether children with early, late and persistent preschool wheezing had lower HRQOL scores at age 4 years, compared to children without preschool wheezing. This study will help to identify the HRQOL domains needing attention in 4 year olds with different wheezing patterns. If our hypothesis is confirmed, this study will highlight the importance of early attention to preschool children's wheezing symptoms and domains of HRQOL.

\section{METHODS}

\section{Design and cohort}

This study is embedded within Generation $R$, a populationbased prospective cohort study [12, 13]. The study was conducted in accordance with the guidelines proposed in the Declaration of Helsinki. The medical ethical committee of the Erasmus MC, University Medical Centre Rotterdam, Rotterdam, The Netherlands, approved this study. Informed consent was obtained from participating parents. Consent for postnatal follow-up was available for 7295 children (fig. 1). Information on wheezing patterns and at least one CHQ-PF28 scale was available for 3878 children (53\% of the postnatal cohort).

\section{Wheezing}

Symptoms of wheezing were assessed by core questions from the International Study of Asthma and Allergies in Childhood at the ages of 1, 2, 3 and 4 years [14]. Response rates for these questionnaires, completed by parents, were $71 \%, 76 \%, 72 \%$ and $73 \%$ respectively. Based on a parentally reported history of wheezing taken from the four questionnaires, preschool children were assigned to the following categories [15, 16]: never wheezing: no wheezing in the first 4 years of life $(n=1996)$; early wheezing: at least one episode of wheezing in the first 3 years and no wheezing in the fourth year $(n=1334)$; late wheezing: no wheezing in the first 3 years and wheezing in the fourth year $(n=95)$ and persistent wheezing: at least one

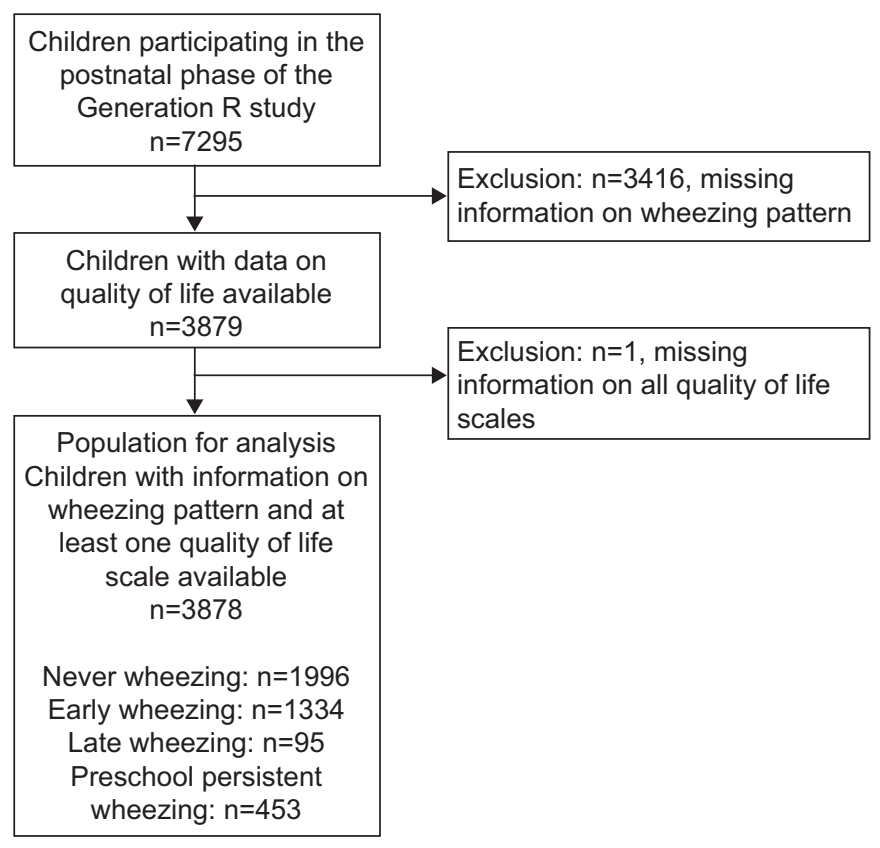

FIGURE 1. Flowchart of participants included for analysis. episode of wheezing in the first 3 years and wheezing in the fourth year $(n=453)$. Additionally, at age 4 years, parental reports on frequency of wheezing (one to three episodes, four or more episodes) were collected [14].

\section{Health-related quality of life}

The CHQ-PF28 was used to measure HRQOL of the child at age 4 years [17]. Based on 28 items, the CHQ-PF28 measures the HRQOL of children and their families across 13 scales (see table 1) $[18,19]$. The following eight multi-item scales measure the child's HRQOL: "physical functioning"; "role functioning: emotional"; "role functioning: physical"; "bodily pain"; "general behaviour"; "mental health"; "self esteem"; "general health perceptions". These multi-item scales are summarised into a "physical summary measure" and a "psychosocial summary measure". Furthermore there is the "change in health" item and the "family cohesion" item. The impact of the child's health on the caregiver's and family's HRQOL is measured across the remaining three multi-item scales: "parental impact: emotional"; "parental impact: time"; "family activities". All scale measures are transformed to scores ranging from 0 to 100. Lower scores correspond to lower HRQOL. Summary measures are standardised with a mean of 50 and standard deviation of 10 to reflect general US population norms for children [18, 19].

\section{Covariates}

The effect of wheezing patterns on children's HRQOL is likely to be influenced by the following covariates. These were selected based on current literature on determinants of HRQOL in children [20, 21].

Maternal characteristics were age, educational level, household income, ethnicity, single motherhood, smoking during pregnancy, atopy and psychopathology. Information about maternal age, the highest attained maternal educational level (low, moderate, high), maternal ethnicity (Dutch, other western, non-western) and single motherhood (yes, no), maternal smoking during pregnancy (yes, no) and maternal atopy (yes, no) were obtained at enrolment in the study by questionnaires. Maternal educational level and maternal ethnicity were defined according to the classifications of Statistics Netherlands [22, 23]. Data on household income $(<€ 1600$ per month, $\geqslant € 1600$ per month) was obtained at the child's age of 3 years, using the 2005 monthly general labour income as the cut-off point [24]. Maternal psychopathology (score in tertiles) was assessed at the child's age of 2 months using the Global Severity Index (GSI) of the Brief Symptom Inventory (a validated self-report measure, which consists of 53 positive and negative self-appraisal statements) [25]. Respective item scores were summed to derive a total score of the GSI (range: 0-200). Total scores were divided into tertiles (cut-off points: three and 10).

Child's characteristics were sex, gestational age at birth, birth weight, exposure to tobacco smoke. Information on sex (boy, girl), gestational age at birth (weeks) and birth weight (grammes), were obtained from medical records. Tobacco smoke exposure (yes, no) was measured at age 2 years, using parental reported questionnaires.

\section{Data analyses}

Characteristics of the study population were calculated and stratified by wheezing pattern. $\mathrm{p}$-values for differences between 
TABLE 1 CHQ-PF28 scales, number of items per scale and score interpretation



Reproduced from $[18,19]$ with permission from the publisher. Total $n=3878$. CHQ-PF28: Child Health Questionnaire Parental Form, including 28 items.

wheezing patterns were calculated by means of the Chi-square test for categorical variables and univariate ANOVA for continuous variables. To investigate the association between wheezing patterns and HRQOL, differences in mean HRQOL scores of early, late and persistent wheezing were compared separately with the mean HRQOL scores of those without preschool wheezing. In order to indicate the relevance of statistically significant differences, effect sizes (d) were calculated by dividing the difference in mean scores between wheezing patterns by the largest standard deviation. Cohen suggests that $d$ values of $0.2,0.5$, and 0.8 respectively represent small, medium, and large effect sizes [26].

Linear regression models were computed with wheezing patterns as the determinant and each of the CHQ-PF28 scales as outcomes. In multivariate linear regression analyses maternal and child's characteristics were added to these models. Additionally, the association between frequency of wheezing in the fourth year of life and HRQOL was studied, using linear regression models. A Bonferroni correction was implemented to account for the number of analyses conducted.
Missing values in the covariates ranged from $0 \%$ (sex) to $19 \%$ (maternal psychopathology). Missing values of the outcome were independent of the exposure and vice versa. Because the missing covariates were not completely at random, completecase analysis was likely to introduce biased results. A multiple imputation method was used to impute missing covariates [27]. 10 imputed datasets were generated using a fully conditional specified model to handle missing values. Imputations were based on the relationships between all variables in the study. No differences in results were observed between analyses with imputed missing data or complete cases.

Analyses were performed using the Statistical Package for Social Sciences (SPSS) version 18.0 for Windows (SPSS Inc., Chicago, IL, USA).

\section{RESULTS}

Complete data on wheezing patterns were available in 3878 children. Table 2 shows the characteristics of the study population, stratified by wheezing pattern. In total $51.5 \%$ $(n=1996)$ of the children never wheezed (the reference group), 
TABLE 2 Characteristics of the study population by preschool wheezing pattern

\begin{tabular}{|c|c|c|c|c|c|c|}
\hline \multirow[t]{2}{*}{ Characteristics } & & \multicolumn{4}{|c|}{ Preschool wheezing pattern } & \multirow[t]{2}{*}{ p-value ${ }^{\#}$} \\
\hline & & Never & Early & Late & Persistent & \\
\hline \multicolumn{7}{|l|}{ Maternal characteristics } \\
\hline Age at enrolment years & & $31.8 \pm 4.3$ & $31.7 \pm 4.3$ & $31.4 \pm 5.1$ & $31.5 \pm 4.9$ & 0.184 \\
\hline Educational level & High & $1264(63.6)$ & $802(61.0)$ & 47 (49.5) & $219(49.4)$ & \\
\hline \multirow[t]{2}{*}{ Household income $€$ per month } & $<1600$ & $209(11.1)$ & $138(11.8)$ & $15(17.4)$ & $85(21.6)$ & $<0.001$ \\
\hline & $\geqslant 1600$ & $1680(88.9)$ & $1029(88.2)$ & 71 (82.6) & $309(78.4)$ & \\
\hline \multirow[t]{3}{*}{ Ethnicity } & Dutch & 1407 (71.2) & $908(69.1)$ & $59(62.8)$ & $253(58.4)$ & \\
\hline & Other western & $237(12.0)$ & $188(14.3)$ & $13(13.8)$ & 85 (19.6) & $<0.001$ \\
\hline & Non-western & $333(16.8)$ & $218(16.6)$ & $22(23.4)$ & $95(21.9)$ & \\
\hline \multirow{2}{*}{ Psychopathology } & Middle tertile & $561(33.0)$ & $334(32.4)$ & 28 (32.9) & $107(31.7)$ & $<0.001$ \\
\hline & Lowest tertile & $689(41.0)$ & $337(32.7)$ & $24(28.2)$ & $83(24.6)$ & \\
\hline \multicolumn{7}{|l|}{ Child's characteristics } \\
\hline Sex & Boy & $903(45.2)$ & $733(54.9)$ & $54(56.8)$ & $254(56.1)$ & $<0.001$ \\
\hline Gestational age weeks & & $40.0 \pm 1.6$ & $39.7 \pm 1.9$ & $40.1 \pm 1.5$ & $39.7 \pm 1.9$ & $<0.001$ \\
\hline Birth weight $g$ & & $3480 \pm 537$ & $3414 \pm 589$ & $3517 \pm 542$ & $3395 \pm 616$ & 0.002 \\
\hline Respiratory tract infections & Yes & $660(34.8)$ & $620(54.2)$ & $34(37.4)$ & $228(60.2)$ & $<0.001$ \\
\hline Postnatal tobacco smoke exposure & Yes & $271(13.7)$ & $191(15.1)$ & $17(17.9)$ & $88(21.0)$ & $<0.001$ \\
\hline Doctor-diagnosed asthma & Yes & $12(0.6)$ & $63(5.1)$ & $1(1.1)$ & $78(19.2)$ & $<0.001$ \\
\hline \multirow[t]{2}{*}{ Frequency of wheezing (fourth year) } & Never & $1996(100)$ & $1334(100)$ & $0(0)$ & $0(0)$ & \\
\hline & $1-3$ times & $0(0)$ & (0) & $78(87.6)$ & $339(79.6)$ & $<0.001$ \\
\hline
\end{tabular}

Data are presented as $n(\%)$ or mean \pm SD. Total $n=3878$. \#: univariate ANOVA for continuous variables and Chi-squared tests for categorical variables. All maternal characteristics, except psychopathology, were obtained at enrolment in the study by questionnaires. Maternal educational level and ethnicity were defined according to the classification of Statistics Netherlands [22, 23]. Household income was obtained at age 3 years [24]. Maternal psychopathology was assessed at child's age 2 months using the Global Severity Index [25]. Child's sex, gestational age at birth and birth weight were obtained from medical records. Using questionnaires, child's respiratory tract infections, tobacco smoke exposure and doctor-diagnosed asthma were measured at the ages of 1,2 and 3 years, respectively.

$34.4 \%(\mathrm{n}=1334)$ wheezed early, $2.4 \%(\mathrm{n}=95)$ wheezed late and $11.7 \%(n=453)$ wheezed persistently during the preschool age. All characteristics in table 2, except maternal age at enrolment, were associated with wheezing patterns $(p<0.05)$. Compared with the reference group, maternal psychopathology $(\mathrm{p}<0.05)$ was different for all wheezing patterns. Compared with the reference group, the proportions of single motherhood $(\mathrm{p}<0.01)$, smoking during pregnancy $(\mathrm{p}<0.05)$, maternal atopy $(\mathrm{p}<0.001)$, gestational age $(\mathrm{p}<0.01)$ and birthweight $(\mathrm{p}<0.01)$ were different for early and persistent wheezing. Children with late and persistent wheezing more often had low maternal educational level (compared to the reference group, $p<0.01$ ). Children with persistent wheezing were more often non-Dutch and from low-income families $(<€ 1600$ per month) compared with the reference group $(\mathrm{p}<0.001)$.

Table 3 shows mean CHQ-PF28 scores at age 4 years for children with early wheezing, late wheezing and persistent wheezing compared to children who never wheezed. Based on parent reports, children with early, late and persistent wheezing had lower scores than the reference group (except for the "change in health" scale). On average, parents of children with early and persistent wheezing reported more often an improvement in health, compared to the reference group $(\mathrm{p}<0.001)$. Relevant differences in CHQ-PF28 scores were found in children with persistent wheezing for the scales: "bodily pain", "general health perceptions", "parental impact (emotional and time)". Most effect sizes were small, except for scale "general health perceptions" in children with persistent wheezing $(\mathrm{d}=0.78)$.

After adjustment for maternal and child's characteristics, children with persistent wheezing had lower scores on all CHQ-PF28 scales, except for "general behaviour", "mental health", "self esteem", "family cohesion" and "change in health". Scores on the "change in health" scale are higher rated in children with early and persistent wheezing compared to children who never wheezed (adjusted regression coefficient $(\mathrm{a} \beta)=4.4,95 \%$ CI $3.1-5.6$ and $a \beta=8.5,95 \%$ CI 6.7-10.4, respectively) (table 4). On the scales "bodily pain" and the 
TABLE 3 CHQ-PF28 scale scores for children with early, late and persistent wheezing compared to children who never wheezed

CHQ-PF28 scales

CHQ PF-28 scale scores

\begin{tabular}{lllllll}
\hline Never wheezing & Early wheezing & $\begin{array}{c}\text { Effect } \\
\text { size }^{\#}\end{array}$ & Late wheezing & $\begin{array}{c}\text { Effect } \\
\text { size }\end{array}$ & $\begin{array}{l}\text { Persistent } \\
\text { wheezing }\end{array}$ & $\begin{array}{c}\text { Effect } \\
\text { size }\end{array}$
\end{tabular}

Subjects $n$

Physical functioning

Role functioning: emotional

Role functioning: physical

Bodily pain

General behaviour

Mental health

Self esteem

General health perceptions

Parental impact: emotional

Parental impact: time

Family activities

Family cohesion

Change in health

Physical summary score

Psychosocial summary score

1996
$98.4 \pm 8.8$
$98.7 \pm 8.1$
$98.1 \pm 10.2$
$88.5 \pm 16.7$
$73.7 \pm 14.0$
$83.9 \pm 13.7$
$83.8 \pm 14.7$
$91.1 \pm 12.1$
$90.1 \pm 13.0$
$94.6 \pm 12.6$
$89.2 \pm 16.3$
$78.0 \pm 17.6$
$56.1 \pm 15.1$
$58.1 \pm 5.6$
$53.8 \pm 6.2$

1996

$98.7 \pm 8.1$

1334

$98.2 \pm 9.6$

$98.2 \pm 9.5^{*}$

$97.8 \pm 10.6$

$88.1 \pm 16.7$

$71.9 \pm 14.3^{\star * *}$

$82.8 \pm 13.4^{* *}$

$83.0 \pm 14.4$

$87.2 \pm 15.2^{* * *}$

$88.6 \pm 13.8^{\star *}$

$93.7 \pm 13.6^{*}$

$87.4 \pm 17.2^{\star \star \star}$

$76.9 \pm 17.7$

$60.9 \pm 18.7^{\star \star *}$

$57.5 \pm 6.0^{* *}$

$53.0 \pm 6.4^{* \star *}$

0.02
0.06
0.03
0.02
0.13
0.08
0.06
0.26
0.11
0.07
0.10
0.06
-0.26
0.10
0.11

95

$96.9 \pm 11.9^{*}$

$97.5 \pm 10.2$

$96.8 \pm 11.0^{*}$

$85.3 \pm 18.4$

$70.7 \pm 14.9^{*}$

$81.5 \pm 13.7$

$82.1 \pm 13.9$

$82.0 \pm 18.9^{* * *}$

$85.3 \pm 19.0^{*}$

$92.1 \pm 16.4$

$85.8 \pm 18.7$

$74.5 \pm 18.4$

$56.4 \pm 18.3$

$55.7 \pm 7.3^{\star \star \star}$

$52.3 \pm 7.1$
453

$94.8 \pm 15.5^{\star * *}$

$96.3 \pm 15.7^{* * *}$

$94.4 \pm 18.2^{* * *}$

$82.6 \pm 18.9^{* * *}$

$71.6 \pm 15.7^{* *}$

$81.9 \pm 14.4^{\star \star}$

$83.5 \pm 14.8$

$76.4 \pm 18.9^{\star \star \star}$

$84.5 \pm 16.6^{\star \star *}$

$89.6 \pm 18.2^{* * *}$

$84.9 \pm 19.2^{\star * *}$

$73.8 \pm 19.4^{\star * *}$

$65.6+21.6^{\star * *}$

$54.0 \pm 8.7^{\star \star \star \star}$

$52.7 \pm 7.2^{\star *}$
0.24

0.16

0.20

0.31

0.14

0.14

0.02

0.78

0.33

0.28

0.22

0.22

$-0.44$

0.47

0.15

Data are presented as mean \pm SD unless otherwise stated. Total $n=3878$. Cohen's effect sizes (d) for differences in Health Related Quality of Life (HRQOL) between preschool wheezing patterns: ${ }^{\#}$ : early wheezing versus never wheezing; ${ }^{\prime}$ : late wheezing versus never wheezing; ${ }^{+}$: persistent wheezing versus never wheezing. ${ }^{*}: p \leqslant 0.05,{ }^{* *}: p \leqslant 0.01,{ }^{* *}: p \leqslant 0.001, p$-values are based on Mann-Whitney U-test [28] for differences between wheezing patterns (never wheezing is the reference group). CHQ-PF28: Child Health Questionnaire Parental Form 28 items.

"physical summary measure", not only parents of children with persistent wheezing, but also parents of children with late wheezing reported significantly poorer HRQOL compared to parents of children who never wheezed. The strongest associations were found for scores on "general health perceptions" in children with early, late and persistent wheezing compared to children who never wheezed $(a \beta=-3.0,95 \%$ CI $-4.0--2.0$; $\mathrm{a} \beta=-7.8,95 \%$ CI $-10.9--4.7$ and $\mathrm{a} \beta=-12.3$, 95\% CI $-13.8-$ -10.7 , respectively). These associations remained statistically significant after applying a Bonferroni correction for multiple testing $(\mathrm{p}<0.003$; i.e. $0.05 / 15)$.

The majority of wheezing children had relative infrequent symptoms (one to three episodes a year) in the fourth year of life and experienced only a limited reduction in HRQOL (online supplementary table S2). Only in the small group with frequent wheezing (more than four episodes a year) did we observe a substantial impact on the child's well-being, particularly on the scores of "general health perceptions" (a $\beta=-23.7,95 \% \mathrm{CI}$ -26.7- -20.7, compared to children without wheezing in the fourth year) as well as on physical domains of HRQOL and parental concerns (effect estimates of "bodily pain": $a \beta=-10.8$, 95\% CI -14.5-7.1 and effect estimates of "parental impact: emotional": $a \beta=-11.6,95 \%$ CI $-14.7--8.6)$.

\section{Non-response analyses}

Excluded children, with missing data on wheezing patterns and HRQOL (n=3417), were compared with included children, who had information on wheezing patterns and HRQOL $(n=3878)$ (online supplementary table S1). Differences were present in all covariates, except for sex, respiratory tract infections and maternal atopy $(\mathrm{p}>0.05)$.

\section{DISCUSSION}

This longitudinal cohort study shows low scores on "general health perceptions" and also on the "physical summary scale" of HRQOL in children with late and persistent wheezing, independent of several maternal and child's characteristics. HRQOL was more affected by frequent wheezing episodes in the fourth year, than by duration of wheezing at age $0-4$ years. No differences in scores on the "psychosocial summary scale" were found between children with different wheezing patterns. Persistent wheezing in preschool children has an impact on the family, affecting the scales "family activities" and "parental impact (emotional and time)". Although most observed effects of early and late wheezing on child's HRQOL are small, an almost large effect $(\mathrm{d}=0.78)$ of persistent wheezing was found on "general health perceptions", already at preschool age. The low scores on "general health perceptions" should be interpreted as a subjective evaluation of a child's general health: parents of children with persistent wheezing believe that their child's health is poor and likely to get worse.

Several studies previously assessed the association between wheezing and HRQOL in childhood $[2,7,8,10]$ and observed that wheezing was associated with poor HRQOL. However, these studies used a cross-sectional design that made it impossible to explore the relative impact of wheezing patterns during preschool age. By using a longitudinal design, our study shows that exposure to wheezing during preschool age affects 
TABLE 4 Crude and adjusted associations between preschool wheezing patterns and the CHQ-PF28 scores at age 4 years



Data are presented as regression coefficients and $95 \%$ confidence intervals estimated by linear regression models. Total $n=3878$; never wheezing group $n=19$; early wheezing $n=1334$; late wheezing $n=95$; persistent wheezing $n=453$. ${ }^{*}$ : based on a parentally reported history of wheezing at age $0-4$ years, children were assigned to the following categories [15, 16]: never, early, late and persistent preschool wheezing. Each wheezing subgroup is compared to children who never wheezed. The crude model shows the association between wheezing patterns and the Child Health Questionnaire Parental Form 28 items (CHQ-PF28) scales, unadjusted for covariates. The adjusted model is adjusted for covariates (including potential confounders): maternal age, maternal educational level and maternal ethnicity, household income, single motherhood, smoking during pregnancy, maternal atopy, maternal psychopathology, child's gender, gestational age and birth weight, child's exact age at measurement of health-related quality of life (HRQOL) and child's exposure to tobacco smoking. Scales are analysed combined using the Physical Summary Score and Psychosocial Summary Score. Bold numbers indicate a significant difference in HRQOL score relative to the reference group. See table 2 for the definition of the scales.

general health perceptions and more specifically affects physical domains of HRQOL at age 4 years. Additionally we found that HRQOL was more affected by frequent wheezing episodes in the fourth year, than by duration of wheezing at age $0-4$ years.

Impairment at age 4 years is most pronounced for nine out of 13 CHQ-PF28 scales in children with persistent wheezing, compared to children who never wheezed. Comparing the associations between preschool wheezing patterns and "physical" and "psychosocial summary measures" (tables 3 and 4), our findings support a previous finding in school-aged children: that a child's asthma particularly impairs the physical domains of HRQOL [2]. Similar to studies in adolescents [29], we also observed that wheezing has an impact on parental perceptions with regard to children's "general health" and "bodily pain" at preschool age. However, we did not observe any impact on "self esteem" or "mental health" [29], suggesting that perhaps the impact emerges after preschool age. The observation that a positive change in health was reported by parents for children with early and persistent wheezing compared to children who never wheezed is not unexpected. These children had previous wheezing symptoms and it is likely that these children were already free of symptoms at the time of completing the questionnaire.

This study benefits from a large sample size and a longitudinal design, which enabled us to classify wheezing symptoms into longitudinal patterns. A prospective design with repeated measurements may be especially important in paediatric asthma research. Recent longitudinal studies have made clear 
that childhood asthma can be highly variable with respect to symptoms as well as time course $[15,16]$.

The results of this study should be viewed in light of several limitations. Mothers of children who were included were higher educated, more healthy and more frequently of Dutch origin than those of children who were excluded. Therefore, selection bias may have occurred, for example if non-participating parents (due to non-response or lost to follow-up) whose children had wheezing symptoms systematically provided higher (or lower) scores on child's HRQOL compared to participating parents whose children had wheezing symptoms. Furthermore, the children in this study may not fully represent the general population as all of them resided in Rotterdam.

Wheezing and HRQOL were measured by parental reports. Parental reports of wheezing are widely accepted in epidemiological studies and reliably reflect the incidence of wheezing in preschool children [30]. When children do not have the cognitive ability to report their own HRQOL, proxy reports by parents are appropriate sources of information about HRQOL [31]. Both overestimation and underestimation of HRQOL scores may have occurred. PETSIOs et al. [31] have shown that parents may tend to overestimate HRQOL of their asthmatic school-aged child. Also it cannot be totally ruled out that current wheezing was associated with increased parental awareness, leading to an underestimation of child's HRQOL. Information about HRQOL was prospectively collected without direct reference to wheezing and we did adjust for relevant parent-related characteristics in our analyses (single parenthood, low educational level, family income and maternal psychopathology) and found that some differences in child's HRQOL between children with different wheezing patterns remained present. Regardless, it is possible that the differences that we found may have been affected by parent-related characteristics other than the ones that we studied [32]. Additional research incorporating child self-report on HRQOL is needed during follow-up at school age to substantiate our findings [33].

We used Cohen's d for the interpretation of relevant differences in HRQOL. Although this is an accepted method, there are still insufficient data to understand the relative impact of the observed score differences. Empirically defined cut-off points for minimal important differences for HRQOL measures such as the CHQ-PF28 are important in future research.

The CHQ-PF28 is a generic HRQOL questionnaire and has the advantage of measuring multiple dimensions of HRQOL across a diversity of conditions to understand the relative impact of diseases and conditions for children and their families. As such, it is possible to compare the HRQOL of children with and without certain symptoms. However, the use of both a general HRQOL questionnaire in concert with a condition-specific measure may further enrich our understanding of the relative and specific impact on children's health and well-being. For example, an asthma-specific measure may provide insight into the specific impact of sleeping problems due to wheezing, while the generic questionnaire may help to position this impact relative to children who may also experience this issue but have not been diagnosed with asthma (e.g. children with attention deficit hyperactivity disorder or cancer).
Although we were able to adjust for important maternal and child characteristics, it should be acknowledged that, in the present study, unmeasured variables, such as detailed information on healthcare use, genetic factors [6] or treatment responsiveness, could (in part) explain the association between wheezing patterns and HRQOL [32].

In conclusion, dynamic patterns of preschool wheezing showed differential effects on HRQOL at age 4 years, independent of several maternal and child's characteristics. Particularly, persistent wheezing during preschool age affects general health perceptions and physical domains at age 4 years. HRQOL was more affected by frequent wheezing episodes in the fourth year, than by duration of wheezing at age $0-4$ years. These findings suggest the need to study how improvement of HRQOL among children with persistent wheezing symptoms might be promoted, with specific attention to the physical domain in children with frequent preschool wheezing.

\section{SUPPORT STATEMENT}

L. Duijts received funding by means of a European Respiratory Society/Marie Curie Joint Research Fellowship (MC 1226-2009) under grant agreement RESPIRE, PCOFUND-GA-2008-229571. V.W.V. Jaddoe received additional grants from the Netherlands Organization for Health Research and Development (ZonMw 90700303 and 916.10159). The general design of the Generation R Study is made possible by financial support from the Erasmus Medical Centre, Rotterdam, the Erasmus University Rotterdam, the Netherlands Organization for Health Research and Development (ZonMw), the Netherlands Organisation for Scientific Research (NWO), the Ministry of Health, Welfare and Sport and the Ministry of Youth and Families.

\section{STATEMENT OF INTEREST}

None declared.

\section{ACKNOWLEDGEMENTS}

The Generation R Study is conducted by the Erasmus Medical Centre, Rotterdam, the Netherlands, in close collaboration with the School of Law and Faculty of Social Sciences of the Erasmus University Rotterdam, the Municipal Health Service Rotterdam area, the Rotterdam Homecare Foundation and the Stichting Trombosedienst and Artsenlaboratorium Rijnmond (STAR-MDC), Rotterdam. We gratefully acknowledge the contribution of the children and parents, general practitioners, hospitals, midwives and pharmacies in Rotterdam.

\section{REFERENCES}

1 Masoli M, Fabian D, Holt S, et al. Global Initiative for Asthma Program. The global burden of asthma: executive summary of the GINA Dissemination Committee report. Allergy 2004; 59: 469-478.

2 Merikallio VJ, Mustalahti K, Remes ST, et al. Comparison of quality of life between asthmatic and healthy school children. Pediatr Allergy Immunol 2005; 16: 332-340.

3 Reddel HK, Taylor DR, Bateman ED, et al. An official American Thoracic Society/European Respiratory Society statement: asthma control and exacerbations: standardizing endpoints for clinical asthma trials and clinical practice. Am J Respir Crit Care Med 2009; 180: 59-99.

4 Pedersen SE, Hurd SS, Lemanske RF, et al. Global strategy for the diagnosis and management of asthma in children 5 years and younger. Pediatr Pulmonol 2011; 46: 1-17.

5 Bateman ED, Bousquet J, Keech ML, et al. The correlation between asthma control and health status: the GOAL study. Eur Respir J 2007; 29: 56-62. 
6 Cortina SD, Drotar D, Ericksen M, et al. Genetic biomarkers of health-related quality of life in paediatric asthma. J Pediatr 2011; 159: 21-26.

7 Sawyer MG, Spurrier N, Kennedy D, et al. The relationship between the quality of life of children with asthma and family functioning. J Asthma 2001; 38: 279-284.

8 Mohangoo AD, Essink-Bot ML, Juniper EF, et al. Health-related quality of life in preschool children with wheezing and dyspnoea: preliminary results from a random general population sample. Qual Life Res 2005; 14: 1931-1936.

9 Mohangoo AD, de Koning HJ, de Jongste JC, et al. Asthma-like symptoms in the first year of life and health-related quality of life at age 12 months: the Generation R study. Qual Life Res 2012; 21: 545-554.

10 Everhart RS, Fiese BH. Asthma severity and child quality of life in paediatric asthma: a systematic review. Patient Educ Couns 2009; 75: $162-168$

11 Caudri D, Wijga AH, Smit HA, et al. Asthma symptoms and medication in the PIAMA birth cohort: evidence for under and overtreatment. Pediatr Allergy Immunol 2011; 22: 652-659.

12 Jaddoe VW, Bakker R, van Duijn CM, et al. The Generation R Study Biobank: a resource for epidemiological studies in children and their parents. Eur J Epidemiol 2007; 22: 917-923.

13 Jaddoe VW, van Duijn CM, van der Heijden AJ, et al. The Generation R Study: design and cohort update 2010. Eur J Epidemiol 2010; 25: 823-841.

14 Sole D, Vanna AT, Yamada E, et al. International Study of Asthma and Allergies in Childhood (ISAAC) written questionnaire: validation of the asthma component among Brazilian children. J Investig Allergol Clin Immunol 1998; 8: 376-382.

15 Brussee JE, Smit HA, Koopman LP, et al. Interrupter resistance and wheezing phenotypes at 4 years of age. Am J Respir Crit Care Med 2004; 169: 209-213

16 Martinez FD, Wright AL, Taussig LM, et al. Asthma and wheezing in the first six years of life. The Group Health Medical Associates. N Engl J Med 1995; 332: 133-138.

17 Raat H, Botterweck AM, Landgraf JM, et al. Reliability and validity of the short form of the child health questionnaire for parents (CHQ-PF28) in large random school based and general population samples. J Epidemiol Community Health 2005; 59: 75-82.

18 Landgraf J, Abetz J, Ware JE. Child Health Questionnaire (CHQ): A User's Manual. Boston, HealthAct, 1999.
19 HealthActCHQ. Child health Questionnaire Scoring and Interpretation Manual. Cambridge, HealthActCHQ Inc., 2008.

20 Jirojanakul P, Skevington SM, Hudson J. Predicting young children's quality of life. Soc Sci Med 2003; 57: 1277-1288.

21 Von Rueden U, Gosch A, Rajmil L, et al. Socioeconomic determinants of health related quality of life in childhood and adolescence: results from a European study. J Epidemiol Community Health 2006; 60: 130-135.

22 Swertz O, Duimelaar P, Thijssen J. Migrants in the Netherlands 2004. Voorburg/Heerlen, Statistics Netherlands, 2004.

23 Statistics Netherlands. The Dutch Standard Classification of Education. Voorburg/Heerlen, Statistics Netherlands, 2004.

24 CPB Netherlands Bureau for Economic Policy Analysis. Beschrijving koopkrachtberekening, CPB Memorandum 133, December 12, 2005. www.cpb.nl/en/publication/beschrijvingkoopkrachtberekening Date last accessed: November 29, 2009.

25 Derogatis LR. Brief Symptom Inventory (BSI): Administration, Scoring and Procedures Manual. Minneapolis, National Computer Systems, 1993.

26 Cohen J. Statistical Power Analysis for the Behavioural Sciences New York, Academy Press, 1988.

27 Greenland S, Finkle WD. A critical look at methods for handling missing covariates in epidemiologic regression analyses. Am J Epidemiol 1995; 142: 1255-1264.

28 Altman D. Practical Statistics for Medical Research. London, Chapman and Hall, 2000.

29 Mohangoo AD, de Koning HJ, Mangunkusumo RT, et al. Healthrelated quality of life in adolescents with wheezing attacks. J Adolesc Health 2007; 41: 464-471.

30 Jenkins MA, Clarke JR, Carlin JB, et al. Validation of questionnaire and bronchial hyperresponsiveness against respiratory physician assessment in the diagnosis of asthma. Int J Epidemiol 1996; 25: 609-616.

31 Petsios K, Priftis KN, Tsoumakas C, et al. Level of parent-asthmatic child agreement on health-related quality of life. J Asthma 2011; 48: 286-297.

32 Theunissen NC, Vogels TG, Koopman HM, et al. The proxy problem: child report versus parent report in health-related quality of life research. Qual Life Res 1998; 7: 387-397.

33 Annett RD, Bender BG, Lapidus J, et al. Predicting children's quality of life in an asthma clinical trial: what do children's reports tell us? J Pediatr 2001; 139: 854-861. 